\title{
Modeling Systems with Internal State using Evolino
}

\author{
Daan Wierstra ${ }^{1}$ \\ daan@idsia.ch
}

\author{
Faustino J. Gomez ${ }^{1}$ \\ tino@idsia.ch
}

\author{
Jürgen Schmidhuber ${ }^{1,2}$ \\ juergen@idsia.ch
}

\author{
1 IDSIA, Galleria 2, 6928 Lugano, Switzerland \\ 2 TU Munich, Boltzmannstr. 3, 85748 Garching, München, Germany
}

\begin{abstract}
Existing Recurrent Neural Networks (RNNs) are limited in their ability to model dynamical systems with nonlinearities and hidden internal states. Here we use our general framework for sequence learning, EVOlution of recurrent systems with LINear Outputs (Evolino), to discover good RNN hidden node weights through evolution, while using linear regression to compute an optimal linear mapping from hidden state to output. Using the Long Short-Term Memory RNN Architecture, Evolino outperforms previous state-of-the-art methods on several tasks: 1) context-sensitive languages, 2) multiple superimposed sine waves.
\end{abstract}

\section{Categories and Subject Descriptors}

I.2.6 [Artificial Intelligence]: Learning-Connectionism and neural nets

\section{General Terms}

Experimentation, Performance, Algorithms

\section{Keywords}

Time-series prediction, Recurrent Neural Networks, Evolution and Learning

\section{INTRODUCTION}

The kinds of sophisticated behaviors we would like artificial agents to perform in the the future will require them to build predictive models of their environment. For example, imagine an active-vision-based robot trying to locate an object in a cluttered space. The robot receives a sequence (time-series) of sensory images from which it decides the most profitable place to look next. To solve this task efficiently, the robot must anticipate the location of the object based on some kind of short-term memory or internal state that helps it determine where it is and where it has been, both spatially and in the sequence sub-goals necessary for

Permission to make digital or hard copies of all or part of this work for personal or classroom use is granted without fee provided that copies are not made or distributed for profit or commercial advantage and that copies bear this notice and the full citation on the first page. To copy otherwise, to republish, to post on servers or to redistribute to lists, requires prior specific permission and/or a fee.

GECCO'05, June 25-29, 2005, Washington, DC, USA.

Copyright 2005 ACM 1-59593-010-8/05/0006 ...\$5.00. accomplishing the task. Otherwise, if the robot does not maintain a useful representation of past experience, it will waste time taking actions that provide no new information about the target object's likely location.

Artificial neural networks are a popular class of models for making predictions from time-series because they are theoretically capable of approximating any continuous mapping with arbitrary precision [4]. Normally, networks are trained using gradient descent, but recently evolutionary computation has been used to either evolve the network structure and then learn the weights via gradient descent (e.g. for Multi-Layer perceptrons [25, 5, 13], and Radial Basis Function Networks (RBF) [21]), or to bypass learning altogether by evolving weight values as well [26]. However, because these methods use feedforward architectures, none of them implement general sequence predictors. That is, predictors that can detect temporal dependencies in the input data that span an arbitrary number of time steps. The MackeyGlass time-series that is often used to test these methods $[21,25,3,5,13]$, for instance, can be predicted very accurately using a feedforward network with a relatively short time-delay window on the input.

Recurrent Neural Networks (RNNs; [20, 18, 23]) can potentially implement general predictors by using feedback connections to maintain internal state. Unfortunately, RNNs are notoriously difficult to train, even using evolution, when the desired network output is a complex function of potentially the entire input history. In this paper, we demonstrate a method called EVOlution of recurrent systems with LINear Outputs (Evolino; [19]), for automatically designing general sequence predictors. The method combines the evolution of RNNs with analytical methods for computing optimal linear mappings. The approach is demonstrated on two tasks that cannot be solved using a finite set of previous inputs (i.e. using feedforward networks): context-sensitive languages, and superimposed sine wave functions.

The next section provides some background on the state of the art in sequence prediction using recurrent neural networks. Section 3 outlines the Evolino algorithm and describes the specific implementation components in more detail. Section 4 present our experiments in the two domains described above, and sections 5 and 6 discuss the approach in a broader context and summarize our conclusions.

\section{RECURRENT NEURAL SEQUENCE PREDICTION}

Training RNNs with standard gradient descent algorithms such as Backpropagation Through Time and Real Time Re- 
current Learning is only practical when a short time window (less than 10 time-steps) is sufficient to predict the correct system output. For longer temporal dependencies, the gradient vanishes as the error signal is propagated back through time so that network weights are never adjusted correctly to account for events far in the past $[2,9]$.

Echo State Networks (ESNs; [10]) deal with temporal dependencies by simply ignoring the gradients associated with hidden neurons. Composed primarily of a large pool of neurons (typically hundreds or thousands) with fixed random weights, ESNs are trained by computing a set of weights analytically from the pool to the output units using fast, linear regression. The idea is that with so many random hidden units, the pool is capable of very rich dynamics that just need to be correctly "tapped" by adjusting the output weights. This simple approach is currently the title holder in the Mackey-Glass time-series benchmark, improving on the accuracy of all other methods by as much as three orders of magnitude [10].

The drawback of ESNs, of course, is that the only truly computationally powerful, nonlinear part of the net does not learn at all. This means that on some seemingly simple tasks, such as generating multiple superimposed sine waves, the method fails. According to our experience, it is also not able to solve a simple context-sensitive grammar task [6]. Moreover, because ESNs use such a large number of processing units, they are prone to overfitting, i.e. poor generalization.

One method that adapts all weights and succeeds in using gradient information to learn long-term dependencies is Long Short-Term Memory (LSTM; [9, 7]). LSTM uses a specialized network architecture that includes memory cells that can sustain their internal activation indefinitely. The cells have input and output gates that learn to open and close at appropriate times either to let in new information from outside and change the state of the cell, or to let activation out to potentially affect other cells or the network's output. The cell structure enables LSTM to use gradient descent to learn dependencies across almost arbitrarily long time spans. However, in cases where gradient information is of little use due to numerous local minima, LSTM becomes less competitive.

An alternative approach to training RNNs is neuroevolution [24]. Instead of using a single neural network, the space of network parameters is searched in parallel using evolutionary computation. This approach has been very effective in solving continuous, partially observable reinforcement learning tasks where the gradient is not directly available, outperforming conventional methods (e.g. Q-learning, SARSA) on several difficult learning benchmarks [15, 8]. However, neuroevolution is rarely used for supervised learning tasks such as time series prediction because it has difficulty fine-tuning solution parameters (e.g. network weights), and because of the prevailing maxim that gradient information should be used when it is available.

Next we describe the Evolino algorithm, which combines elements of the three methods discussed in this section, to address the disadvantages of each.

\section{THE EVOLINO ALGORITHM}

Evolino is a general framework for temporal sequence prediction that combines neuroevolution and traditional methods for computing optimal linear mappings, such as linear

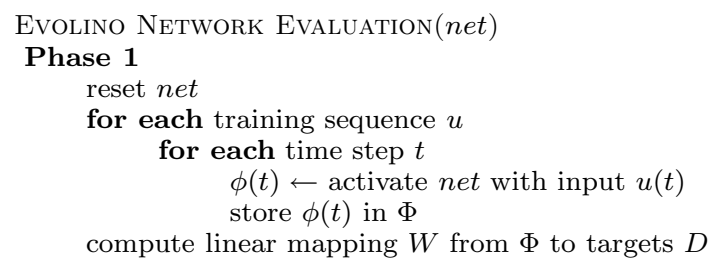

Figure 1: Evolino Network Evaluation. Evolino networks are evaluated in two phases. In the first, the network is fed the training sequences, and the activation pattern of the network is saved at each time step. At this point the network does not have connections to its outputs with which to make predictions. Once the entire training set has been seen, the output weights are calculated analytically, and the training set is seen again, but now the network produces outputs. The error between these predictions and the correct (target) values is used as a fitness score to be minimized by genetic search.

regression. The underlying principle of Evolino is that often a linear model can account for a large number of properties of a problem. The non-linear properties that are not predicted by the linear model, are then dealt with by evolution.

An Evolino network consists of two parts: (1) a non-linear recurrent subnetwork that receives external input, and (2) a linear layer that maps the state of the subnetwork to the output units. The weights of recurrent part of the network are evolved, while the weights of the output layer are computed analytically when the recurrent subnetwork is evaluated during evolution. This procedure generalizes ideas from Maillard [12], in which a similar hybrid approach was used train feedforward networks of radial basis functions.

Network evaluation proceeds in two phases (figure 1). In the first phase, a training set of sequences obtained from the system, $\left\{u^{i}, d^{i}\right\}, i=1 . . k$, each of length $l^{i}$, is presented to the network. For each sequence $u^{i}$, starting at time $t=0$, each input pattern $u^{i}(t)$ is successively propagated through the recurrent subnetwork to produce a vector $\phi^{i}(t)$ that is stored in a $n \times \sum_{i}^{k} l^{i}$ matrix $\Phi$. Associated with each $\phi^{i}(t)$, is a target vector $d^{i}$ in $D$ containing the correct output values for each time step. Once all $k$ sequences have been seen, the output weights $W$ are computed using linear regression from $\Phi$ to $D$. The vectors in $\Phi$ (i.e. the values of each of the $n$ outputs over the entire training set) form a non-orthogonal basis that is combined linearly by $W$ to approximate $D$.

In the second phase, the training set is presented to the network again, but now the inputs are propagated through the recurrent subnetwork and the newly computed output connections to produce predictions $y(t)$. The error in the prediction or the residual error is then used as the fitness measure to be minimized by evolution. 


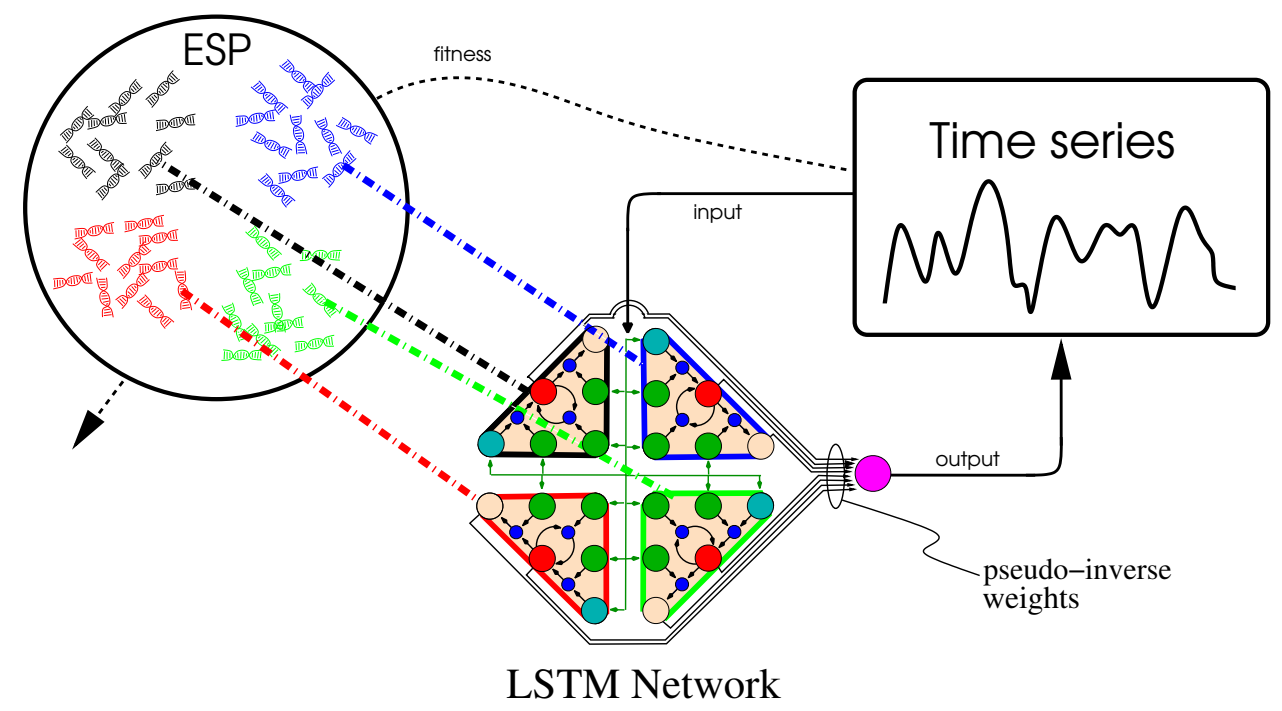

Figure 2: Enforced SubPopulations (ESP). ESP evolves neurons instead of full networks. Neurons are segregated into subpopulations, and networks are formed by randomly selecting one neuron from each subpopulation. A neuron accumulates a fitness score by adding the fitness of each network in which it participated. The best neurons within each subpopulation are mated to form new neurons. The network shown here is an LSTM network with four memory cells. In Evolino, only the connection weights in the recurrent part of the network are evolved. The weights to the output units are computed analytically during each evaluation.

Neuroevolution is normally applied to reinforcement learning tasks where correct network outputs (i.e. targets) are not known a priori. Here we use neuroevolution for supervised learning to circumvent the problem of vanishing gradient. In order to obtain the precision required for time-series prediction, we do not try to evolve a network that makes predictions directly. Instead, the network outputs a set of vectors that form a basis for linear regression. The intuition is that finding a sufficiently good basis is easier than trying to find a network that models the system accurately on its own.

The next sections describe the particular instantiation of Evolino used in this study.

\subsection{Enforced SubPopulations}

Enforced SubPopulations differs from standard neuroevolution methods in that instead of evolving complete networks, it coevolves separate subpopulations of network components or neurons (figure 2). Evolution in ESP proceeds as follows:

1. Initialization. The number of hidden units $H$ in the networks that will be evolved is specified and a subpopulation of $n$ neuron chromosomes is created for each hidden unit. Each chromosome encodes a neuron's input, output, and recurrent connection weights with a string of random real numbers.

2. Evaluation. A neuron is selected at random from each of the $H$ subpopulations, and combined to form a recurrent network. The network is evaluated on the task and awarded a fitness score. The score is added to the cumulative fitness of each neuron that participated in the network.

3. Recombination. For each subpopulation the neurons are ranked by fitness, and the top quartile is recombined using 1-point crossover and mutated using Cauchy distributed noise to create new neurons that replace the lowest-ranking half of the subpopulation.

4. Repeat the Evaluation-Recombination cycle until a sufficiently fit network is found.

ESP searches the space of networks indirectly by sampling the possible networks that can be constructed from the subpopulations of neurons. Network evaluations serve to provide a fitness statistic that is used to produce better neurons that can eventually be combined to form a successful network. This cooperative coevolutionary approach is an extension to Symbiotic, Adaptive Neuroevolution (SANE; [15]) which also evolves neurons, but in a single population. By using separate subpopulations, ESP accelerates the specialization of neurons into different sub-functions needed to form good networks because members of different evolving sub-function types are prevented from mating. Subpopulations also reduce noise in the neuron fitness measure because each evolving neuron type is guaranteed to be represented in every network that is formed. This allows ESP to evolve recurrent networks, where SANE could not.

If the performance of ESP does not improve for a predetermined number of generations, a technique called burst mutation is used. The idea is to search for good modifications of the current best solution. When performance has stagnated for a predetermined number of generations, new subpopulations are created by adding noise to each of the neurons in the best solution. Each new subpopulation contains neurons that represent differences from the best solution. Evolution then resumes, but now searching the space in a "neighborhood" around the best previous solution. Burst mutation can be applied multiple times, with successive invocations representing differences to the previous best solution. Assuming the best solution already has some competence in the task, most of its weights will not 


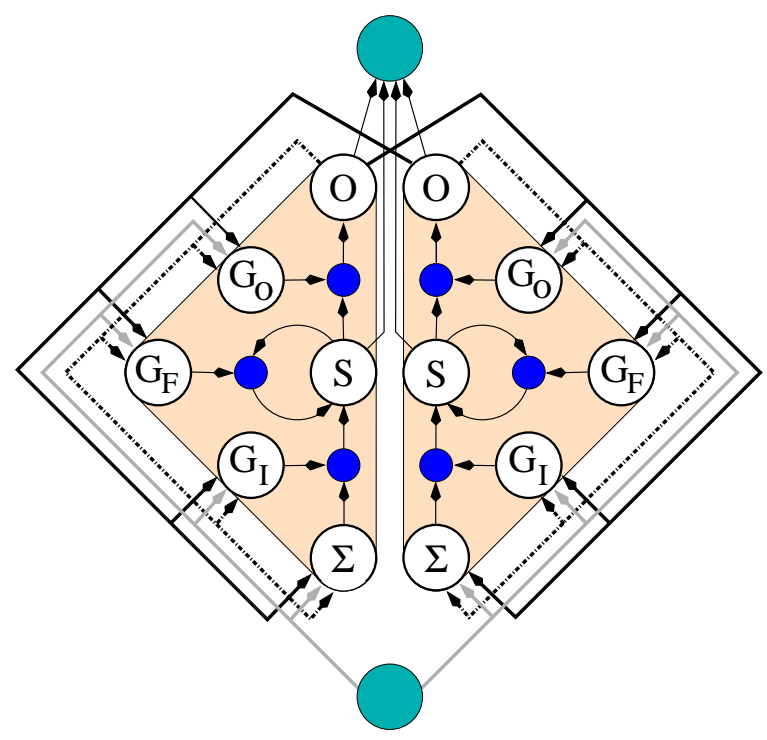

Figure 3: Long Short-Term Memory. The figure shows an LSTM network with one external input (lower-most circle), one output (uppermost circle), and two memory cells (two triangular regions). Each cell has an internal state $S$ together with a forget gate $\left(G_{F}\right)$ that determines how much the state is attenuated at each time step. The input gate $\left(G_{I}\right)$ controls access to the state by the external inputs and the output of other cells which are summed into each $\Sigma$ unit, and the output gate $\left(G_{O}\right)$ controls when and how much the cell fires. Small dark nodes represent the multiplication function.

need to be changed radically. To ensure that most changes are small while allowing for larger changes to some weights, ESP uses the Cauchy distribution to generate noise:

$$
f(x)=\frac{\alpha}{\pi\left(\alpha^{2}+x^{2}\right)}
$$

With this distribution $50 \%$ of the values will fall within the interval $\pm \alpha$ and $99.9 \%$ within the interval $318.3 \pm \alpha$. This technique of "recharging" the subpopulations keeps diversity in the population so that ESP can continue to make progress toward a solution even in prolonged evolution.

Burst mutation is similar to the Delta-Coding technique of Whitley [22] which was developed to improve the precision of genetic algorithms for numerical optimization problems.

\subsection{Long Short-Term Memory}

LSTM is a recurrent neural network purposely designed to learn long-term dependencies via gradient descent. The unique feature of the LSTM architecture is the memory cell that is capable of maintaining its activation indefinitely (figure 3). Memory cells consist of a linear unit which holds the state of the cell, and three gates that can open or close over time. The input gate "protects" a neuron from its input: only when the gate is open, can inputs affect the internal state of the neuron. The output gate lets the internal state out to other parts of the network, and the forget gate enables the state to "leak" activity when it is no longer useful. The gates also receive inputs from neurons, and a function over their input (usually the sigmoid function) decides whether they open or close.
The amount each gate $g_{i}$ of memory cell $i$ is open or closed at time $t$ is calculated by:

$$
\begin{aligned}
g_{i}^{\text {in }}(t) & =\sigma\left(\sum_{j} w_{i j}^{\text {in }} c_{j}(t-1)+\sum_{k} w_{i k}^{\text {in }} u_{k}(t)\right) \\
g_{i}^{\text {forget }}(t) & =\sigma\left(\sum_{j} w_{i j}^{\text {forget }} c_{j}(t-1)+\sum_{k} w_{i k}^{\text {forget }} u_{k}(t)\right) \\
g_{i}^{\text {out }}(t) & =\sigma\left(\sum_{j} w_{i j}^{\text {out }} c_{j}(t-1)+\sum_{k} w_{i k}^{\text {out }} u_{k}(t)\right)
\end{aligned}
$$

where $w_{i j}^{\{\text {in,out,forget }\}}$ is the weight from the output $c_{j}$ of cell $j$ to gate $i, w_{i k}^{\{\text {in,out,forget }\}}$ is the weight from external input $u_{k}$ to the gate $i$, and $\sigma$ is the standard sigmoid function.

The external inputs to the cell (indicated by the $\Sigma$ s in figure 3) are added up in net $_{i}(t)$ :

$$
\operatorname{net}_{i}(t)=h\left(\sum_{j} w_{i j}^{\text {cell }} c_{j}(t-1)+\sum_{k} w_{i k}^{\text {cell }} u_{k}(t)\right),
$$

where $h$ is usually the identity function. The internal state of cell $i$ is:

$$
s_{i}(t)=\operatorname{net}_{i}(t) g_{i}^{i n}(t)+g_{i}^{\text {forget }}(t) s_{i}(t-1),
$$

and the output gates control the cell outputs $c_{i}$ which is squashed by the tanh function:

$$
c_{i}(t)=\tanh \left(g_{i}^{\text {out }}(t) s_{i}(t)\right) .
$$

\subsection{Combining ESP and LSTM in Evolino}

We apply our general Evolino framework to the LSTM architecture, using ESP for evolution and regression for computing linear mappings from hidden state to outputs. ESP coevolves subpopulations of memory cells instead of standard recurrent neurons (figure 2). Each chromosome is a string containing the external input weights and the input, output, and forget gate weights, for a total of $4 *(I+H)$ weights in each memory cell chromosome, where $I$ is the number of external inputs and $H$ is the number of memory cells in the network. There are four sets of $I+H$ weights because the three gates (equations 2,3, and 4) and the cell itself (equation 5) receive input from outside the cell and the other cells. Figure 4 shows how the memory cells are encoded in an ESP chromosome. Each chromosome in a subpopulation encodes the connection weights for a cell's input, output, and forget gates, and external inputs.

ESP, as described in section 3.1, normally uses crossover to recombine neurons. However, for the present Evolino variant, where fine local search is desirable, ESP uses only mutation. The top quarter of the chromosomes in each subpopulation are duplicated and the copies are mutated by adding Cauchy noise (equation 1) to all of their weight values.

The linear regression method used to compute the output weights $W$ is the Moore-Penrose pseudo-inverse method [17], which is both fast and optimal in the sense that it minimizes the summed squared error. For LSTM networks, the vector $\phi(t)$, which is used to compute the output layer weights, consists of both the cell outputs, $c_{i}$ (equation 7 ), and their internal states, $s_{i}$ (equation 6). Therefore, the pseudo-inverse computes two connection weights for each memory cell (the four connections to the output unit in figure 3 ). We refer to the connections from internal states to the output units as "output peephole" connections, since they peer into the 


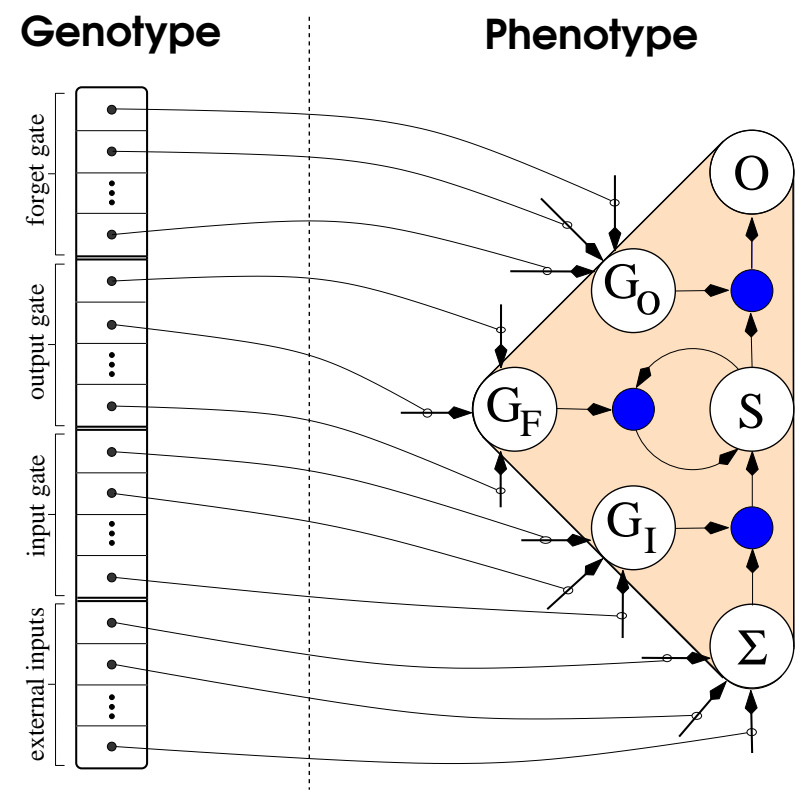

Figure 4: Genotype-Phenotype mapping. Each chromosome (genotype, left) in a subpopulation encodes the external input, and input, output, and forget gate weights of an LSTM memory cell (right). The weights leading out of the state (S) and output (O) units in figure 3 are not encoded in the genotype, but are instead computed at evaluation time by linear regression.

interior of the cells. The output of the network is then:

$$
y_{i}(t)=\sum_{j} c_{j}(t) w_{i j}^{\text {standard }}+\sum_{k} s_{k}(t) w_{i k}^{\text {peephole }} .
$$

For continuous function generation, backprojection is used where the predicted outputs are fed back as inputs in the next time step. During training, the correct target values are backprojected, in effect "clamping" the network's outputs to the right values. During testing, the network backprojects its own predictions. This technique is also used by ESNs, but whereas ESNs do not change the backprojection connection weights, Evolino evolves them, treating them like any other input to the network. In the experiments described below, backprojection was found useful for continuous function generation tasks, but interferes to some extent with performance in the discrete context-sensitive language task.

\section{EXPERIMENTAL RESULTS}

We carried out experiments on two very different domains: continuous function generation and context-sensitive languages. This choice was made to ensure that Evolino did not only perform well in continuous time series prediction, but also on discrete tasks, since many real world applications have both discrete and continuous elements.

To demonstrate the generality of Evolino, we used exactly the same parameters for both tasks, despite their very different characteristics. A bias was added to the forget gates and output gates: +1.5 for the forget gates and -1.5 to the output gates. This did not affect the overall results of the experiments, but sped up learning, especially during the first few generations. The initial weights for the

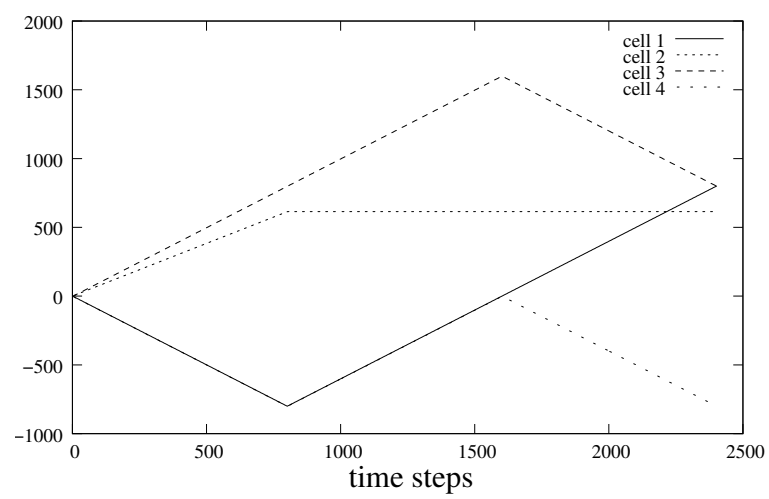

Figure 5: Internal state activations. The state activations for the 4 memory cells of an Evolino network being presented the string $a^{800} b^{800} c^{800}$. The plot clearly shows how some units function as "counters," recording how many $a$ s and $b$ s have been seen.

memory cells were chosen at random from $[-0.1,0.1]$, and the Cauchy noise parameter $\alpha$ both for recombination and burst mutation was set to 0.00001 .

The only difference between the experimental settings of both experiments was the use of backprojection for the superimposed sine wave task. Again, adding backprojection to the language task only slows down evolution but does not qualitatively produce worse results.

\subsection{Context-Sensitive Grammars}

Context-sensitive languages are languages that cannot be recognized by deterministic finite-state automata, and are therefore more complex in some respects than regular languages. In general, determining whether a string of symbols belongs to a context-sensitive language requires remembering all the symbols in the string seen so far, which rules out the use of non-recurrent neural architectures. To compare Evolino-based LSTM with published results for Gradientbased LSTM [6], we chose the language $a^{n} b^{n} c^{n}$.

The task was implemented using networks with 4 input units, one for each symbol $(a, b, c)$ plus the start symbol $S$, and four output units, one for each symbol plus the termination symbol $T$. Symbol strings were presented sequentially to the network, with each symbol's corresponding input unit set to 1 , and the other three set to -1 . At each time step, the network must predict the possible symbols that could come next in a legal string. Legal strings in $a^{n} b^{n} c^{n}$ are those in which the number of $a \mathrm{~s}, b \mathrm{~s}$, and $c \mathrm{~s}$ is equal, e.g. $S T, S a b c T$, $S a a b b c c T$, SaaabbbcccT, and so forth. So, for $n=3$, the set of input and target values would be:

\begin{tabular}{|c|c|c|c|c|c|c|c|c|c|c|}
\hline Input: & $\mathrm{S}$ & $\mathrm{a}$ & $\mathrm{a}$ & $\mathrm{a}$ & $\mathrm{b}$ & $\mathrm{b}$ & $\mathrm{b}$ & $\mathrm{c}$ & $\mathrm{c}$ & $\mathrm{c}$ \\
\hline \hline Target: & $\mathrm{a} / \mathrm{T}$ & $\mathrm{a} / \mathrm{b}$ & $\mathrm{a} / \mathrm{b}$ & $\mathrm{a} / \mathrm{b}$ & $\mathrm{b}$ & $\mathrm{b}$ & $\mathrm{c}$ & $\mathrm{c}$ & $\mathrm{c}$ & $\mathrm{T}$ \\
\hline
\end{tabular}

Evolino-based LSTM networks were evolved using 8 different training sets, each containing legal strings with values for $n$ as shown in the first column of table 1. In the first four sets, $n$ ranges from 1 to $k$, where $k=10,20,30,40$. The second four sets consist of just two training samples, and were intended to test how well the methods could induce the language from a nearly minimal number of examples.

Table 1 compares the results of Evolino-based LSTM re- 


\begin{tabular}{|c|c|c|c|}
\hline $\begin{array}{c}\text { Training } \\
\text { data }\end{array}$ & $\begin{array}{c}\text { Standard } \\
\text { Evolino-LSTM }\end{array}$ & $\begin{array}{c}\text { Tuned } \\
\text { Evolino-LSTM }\end{array}$ & $\begin{array}{c}\text { Gradient- } \\
\text { LSTM }\end{array}$ \\
\hline \hline $1 . .10$ & $1 . .29$ & $1 . .53$ & $1 . .28$ \\
\hline $1 . .20$ & $1 . .67$ & $1 . .95$ & $1 . .66$ \\
\hline $1 . .30$ & $1 . .93$ & $1 . .355$ & $1 . .91$ \\
\hline $1 . .40$ & $1 . .101$ & $1 . .804$ & $1 . .120$ \\
\hline \hline 10,11 & $4 . .14$ & $3 . .35$ & $10 . .11$ \\
\hline 20,21 & $13 . .36$ & $5 . .39$ & $17 . .23$ \\
\hline 30,31 & $26 . .39$ & $3 . .305$ & $29 . .32$ \\
\hline 40,41 & $32 . .53$ & $1 . .726$ & $35 . .45$ \\
\hline
\end{tabular}

Table 1: Results for the $a^{n} b^{n} c^{n}$ language. The table compares Evolino-based LSTM with Gradient-based LSTM on the $a^{n} b^{n} c^{n}$ language task. "Standard" refers to Evolino with the parameter settings used for both discrete and continuous domains $\left(a^{n} b^{n} c^{n}\right.$ and superimposed sine waves). The "Tuned" version is biased to the language task: we additionally squash the cell input with the tanh function. The leftmost column shows the set of strings used for training in each of the experiments. The other three columns show the set of legal strings to which each method could generalize after 50 generations (3000 evaluations), averaged over 20 runs. The upper training sets contain all strings up to the indicated length. The lower training sets only contain a single pair. Evolino-based LSTM generalizes better than Gradientbased LSTM, most notably when trained on only two examples of correct behavior. The Gradient-based LSTM results are taken from [6].

sults with those of Gradient-based LSTM from [6]. "Standard Evolino" uses parameter settings that are a compromise between discrete and continuous domains. If we set $h$ in equation 5 to the tanh function, we obtain "Tuned Evolino."

The Standard Evolino networks had generalization very similar to that of Gradient-based LSTM on the 1..k training sets, but slightly better on the two-example training sets. Tuned Evolino showed a dramatic improvement over Gradient-based LSTM on all of the training sets, but, most remarkably on the two-example sets where it was able to generalize on average to all strings up to $n=726$ after being trained on only $n=\{40,41\}$. Figure 5 , shows the internal states of each of the 4 memory cells of one of the evolved networks while processing $a^{800} b^{800} c^{800}$.

\subsection{Multiple Superimposed Sine Waves}

Learning to generate a sinusoidal signal is a relatively simple task that requires only one bit of memory to indicate whether the current network output is greater or less than the previous output. When sine waves with frequencies that are not integer multiples of each other are superimposed, the resulting signal becomes much harder to predict because its wavelength can be extremely long, i.e. there are large number of time steps before the periodic signal repeats. Generating such a signal accurately without recurrency would require a prohibitively large time-delay window using a feedforward architecture.

Jaeger reports [11] that Echo State Networks are unable to learn functions composed of even two superimposed oscillators, in particular $\sin (0.2 x)+\sin (0.311 x)$. The reason for this is that the dynamics of all the neurons in the ESN

\begin{tabular}{|c|c|c|c|}
\hline No. sines & No. cells & Training error & Gen. error \\
\hline 2 & 10 & 0.0008 & 0.0034 \\
\hline 3 & 15 & 0.0018 & 0.0195 \\
\hline 4 & 20 & 0.0893 & 4.73 \\
\hline 5 & 20 & 0.125 & 13.4 \\
\hline
\end{tabular}

Table 2: Results for multiple superimposed sine waves. The table shows the number of memory cells, training error, and generalization error for each of the superimposed sine wave functions. The training error is the sum of squares error on time steps 100 to 400 (i.e. the washout time is not included in the measure). The generalization error is calculated for time steps 400 to 700 . The error values are the average over 20 experiments.

"pool" are coupled, while this task requires that the two underlying oscillators be represented independently by the network's internal state.

Here we show how Evolino-based LSTM not only can solve the two-sine function mentioned above, but also more complex functions formed by adding up to three more sine waves. The following table shows the four functions used in our experiments, starting with the two-sine suggested by Jaeger [11]. Each of the functions we used was construct by $\sum_{i=1}^{n} \sin \left(\lambda_{i} x\right)$, where $n$ is the number of sine waves and $\lambda_{1}=0.2, \lambda_{2}=0.311, \lambda_{3}=0.42, \lambda_{4}=0.51$, and $\lambda_{5}=0.74$.

Evolino used the same parameter settings as in the previous section, except that backprojection was used (see section 3.3). Networks were evolved to predict, without any external input, the first 400 time steps of each function, using a "washout time" of 100 steps. During the washout time the vectors $\phi(t)$ are not collected for calculating the pseudoinverse. After a specified number of generations, the best networks were then tested for generalization on data points from time-steps 400..700. The first three tasks, $n=2,3,4$, used subpopulations of size 40 and simulations were run for 50 generations. The five-sine wave task, $n=5$, proved much more difficult to learn requiring a larger subpopulation size of 100 , and simulations were allowed to run for 150 generations.

Table 2 shows the number of memory cells used for each task, and the average error on both the training set and the testing set for the best network found during each evolutionary run. Figure 6 shows the behavior of one the successful networks from each of the tasks. The column on the left shows the target signal from Table 2, and the output generated by the network on the training set. The column on the right shows the same curves forward in time to show the generalization capability of the networks. For the two-sine function, even after 9000 time-step, the network continues to generated the signal accurately. As more sines are added, the prediction error grows more quickly, but the overall behavior of the signal is still retained, showing that the network has succeeded in representing the signal's underlying attractors.

\section{DISCUSSION}

The two sequence prediction tasks investigated in this paper demonstrate that Evolino-based LSTM networks are capable of learning both discrete and continuous time-series that require extracting and retaining information from el- 


\section{Training}
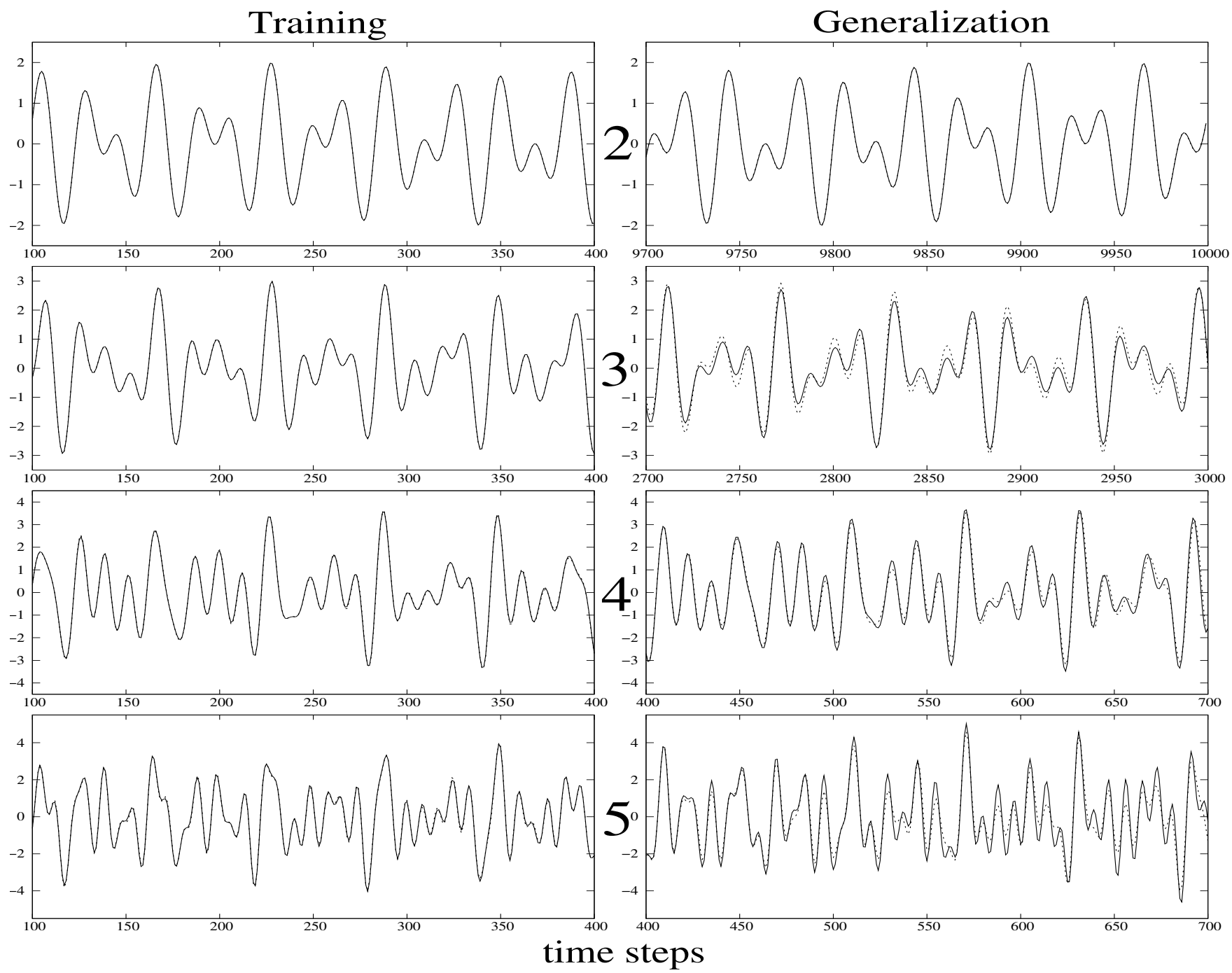

Figure 6: Performance of Evolino-based LSTM on the superimposed sine wave tasks. The plots show the behavior of a typical network produced after a specified number of generations: 50 for the two-, three-, and four-sine functions, and 150 for the five-sine function. The first 300 steps of each function, in the left column, were used for training. The curves in the right column show values predicted by the networks (dashed curves) further into the future vs. the corresponding reference signal (solid curves). While the onset of noticeable prediction error occurs earlier as more sines are added, the networks still track the correct behavior for hundreds of time steps, even for the five-sine function.

ements in the series that can be far in past. This general ability to base decisions on past experience is essential for building robust anticipatory systems that can function in uncertain real-world environments.

On the context-sensitive grammar task, Evolino outperformed Gradient-based LSTM - the only RNN architecture previously able to learn this task. And, it was able capture the underlying dynamics of up to five superimposed sine waves, while Echo State Networks are unable to cope with even the two-sine case. The very different nature of these two tasks suggests that Evolino could be widely applicable to modeling complex processes, such as speech, that have both discrete and continuous properties.

Although Evolino does not use learning in the traditional gradient-descent sense, it is related to other hybrid evolutionary methods that adapt weight values during interaction with the evaluation environment $[16,25,14]$. The "on the fly" computation of the output layer can be viewed as a kind of learning, and, like learning, it effectively distorts the fitness landscape in a Baldwinian sense [1].

The version Evolino used in this paper is but one possible instantiation of the general framework. Many others are possible. For instance, ESP could be replaced by a neuroevolution method that evolves topology as well, or the genetic search, in general, could be complemented by local search, or even gradient-descent. Also, other network architectures could be evolved, such as Higher-Order networks.

Future work will begin by exploring this space of possible implementations further, with a view toward applying them to autonomous mobile robot tasks. Evolino will be used to build forward models that not only can enable a robot to forecast future states and thereby behave more intelligently, 
but also to accelerate the evolution of robot controllers. An Evolino-based model that captures the dynamic elements of an environment that are salient for a particular robot task, can serve as a computationally efficient surrogate for the real-world in which to evaluate candidate controllers.

\section{CONCLUSION}

In this paper, we demonstrated an implementation of EVOlution of recurrent systems with LINear Outputs (Evolino) that used the Enforced SubPopulations neuroevolution algorithm to coevolve Long Short-Term Memory cells. The approach was evaluated on two different prediction tasks that require short-term memory: a discrete problem, contextsensitive languages, and a continuous time-series, multiple superimposed sine waves. In both cases, our results improved upon the state of the art, demonstrating that the method is a powerful and general sequence prediction strategy.

\section{Acknowledgments}

This research was partially funded by CSEM Alpnach and the EU MindRaces project, FP6 511931.

\section{REFERENCES}

[1] J. M. Baldwin. A new factor in evolution. The American Naturalist, 30:441-451, 536-553, 1896.

[2] Y. Bengio, P. Simard, and P. Frasconi. Learning long-term dependencies with gradient descent is difficult. IEEE Transactions on Neural Networks, 5(2):157-166, 1994.

[3] S. Chen. Combined genetic algorithm optimization and regularized orthogonal least squares learning for radial basis function networks. IEEE Transactions on Neural Networks, 10(5), September 1999.

[4] G. Cybenko. Approximation by superpositions of a sigmoidal function. Mathematics of Control, Signals, and Systems, 2:303-314, 1989.

[5] I. D. Falco, A. Iazzetta, P. Natale, and E. Tarantino. Evolutionary neural networks for nonlinear dynamics modeling. In Parallel Problem Solving from Nature 98, volume 1498 of Lectures Notes in Computer Science, pages 593-602, 1998.

[6] F. A. Gers and J. Schmidhuber. LSTM recurrent networks learn simple context free and context sensitive languages. IEEE Transactions on Neural Networks, 12(6):1333-1340, 2001.

[7] F. A. Gers, J. Schmidhuber, and F. Cummins. Learning to forget: Continual prediction with LSTM. Neural Computation, 12(10):2451-2471, 2000.

[8] F. Gomez and R. Miikkulainen. Solving non-Markovian control tasks with neuroevolution. In Proceedings of the 16th International Joint Conference on Artificial Intelligence, Denver, CO, 1999. Morgan Kaufmann.

[9] S. Hochreiter and J. Schmidhuber. Long short-term memory. Neural Computation, 9(8):1735-1780, 1997.

[10] H. Jaeger. Harnessing nonlinearity: Predicting chaotic systems and saving energy in wireless communication. Science, 304:78-80, 2004.
[11] H. Jaeger. http://www.faculty.iu-bremen.de/ hjaeger/courses/seminarspring04/esnstandardslides.pdf, 2004.

[12] E. P. Maillard and D. Gueriot. RBF neural network, basis functions and genetic algorithms. In IEEE International Conference on Neural Networks, pages 2187-2190, Piscataway, NJ, 1997. IEEE.

[13] H. A. Mayer and R. Schwaiger. Evolutionary and coevolutionary approaches to time series prediction using generalized multi-layer perceptrons. In Congress on Evolutionary Computation, Washington D.C., July 1999.

[14] P. McQuesten and R. Miikkulainen. Culling and teaching in neuro-evolution. In T. Bäck, editor, Proceedings of the Seventh International Conference on Genetic Algorithms (ICGA-97, East Lansing, MI), pages 760-767. San Francisco, CA: Morgan Kaufmann, 1997.

[15] D. E. Moriarty and R. Miikkulainen. Efficient reinforcement learning through symbiotic evolution. Machine Learning, 22:11-32, 1996.

[16] S. Nolfi, J. L. Elman, and D. Parisi. Learning and evolution in neural networks. Adaptive Behavior, 2:5-28, 1994.

[17] R. Penrose. A generalized inverse for matrices. In Proceedings of the Cambridge Philosophy Society, volume 51, pages 406-413, 1955.

[18] A. J. Robinson and F. Fallside. The utility driven dynamic error propagation network. Technical Report CUED/F-INFENG/TR.1, Cambridge University Engineering Department, 1987.

[19] J. Schmidhuber, D. Wierstra, and F. Gomez. Evolino: Hybrid neuroevolution/optimal linear search for sequence learning. In Proceedings of the 19th International Joint Conference on Artificial Intelligence, 2005.

[20] P. Werbos. Backpropagation through time: what does it do and how to do it. In Proceedings of IEEE, volume 78, pages 1550-1560, 1990.

[21] B. Whitehead and T. D. Choate. Cooperative-Competitive genetic evolution of radial basis function centers and widths for time series prediction. IEEE Transactions on Neural Networks, 7(4):869-880, 1996

[22] D. Whitley, K. Mathias, and P. Fitzhorn. Delta-Coding: An iterative search strategy for genetic algorithms. In R. K. Belew and L. B. Booker, editors, Proceedings of the Fourth International Conference on Genetic Algorithms, pages 77-84. San Francisco, CA: Morgan Kaufmann, 1991.

[23] R. J. Williams and D. Zipser. A learning algorithm for continually running fully recurrent networks. Neural Computation, 1(2):270-280, 1989.

[24] X. Yao. Evolving artificial neural networks. Proceedings of the IEEE, 87(9):1423-1447, 1999.

[25] X. Yao and Y. Liu. A new evolutionary system for evolving artificial neural networks. IEEE Transactions on Neural Networks, 8(3):694-713, May 1997.

[26] B.-T. Zhang, P. Ohm, and H. Mhlenbein. Evolutionary induction of sparse neural trees. Evolutionary Computation, 5(2):213-236, 1997. 